\title{
PerformanCE ANALYSis OF ROUTING PROTOCOLS WITH ROADSIDE UNIT INFRASTRUCTURE IN A VEHICULAR AD HOC NETWORK
}

\author{
Safae Smiri, Abdelali Boushaba, Rachid Ben Abbou and Azeddine Zahi \\ Intelligent Systems and Applications Laboratory (LSIA), Faculty of Science and \\ Technology, Sidi Mohamed Ben Abdelah University, Fez, Morocco.
}

\begin{abstract}
Vehicular ad-hoc networks (VANETs) represent a powerful and active field of research and have given rise to many challenges related to routing protocols and communication problems with other vehicles or fixed infrastructure called roadside units (RSU). The dynamic topology and the obstacles encountered in VANET environments mean that the routing of data and the communication between vehicles is confronted with many problems, and particularly in vehicular applications that require reliable communication and satisfactory quality of service $(Q o S)$. This paper promotes the intention of infrastructure in an urban scenario and studies the performance of routing protocols considering the constraint of mobility. This leads us to analyze a wide range of routing protocols to ensure optimal coverage and continuous connectivity, taking into consideration two types of data traffic in realistic environments that depend on certain performance metrics. The paper also investigates which protocols provide better performance with $R S U$ s by ranking the results for QoS.
\end{abstract}

\section{KEYWORDS}

VANET, Intelligent Transportation System (ITS); Routing protocols, Roadside unit (RSU), Urban topology, NS-2, VanetMobiSim, Quality of service $(Q o S)$.

\section{INTRODUCTION}

Over the last decade, intelligent transportation systems (ITSs) [1] have aimed to increase our knowledge of traffic and mobility management by using information technologies and communication for sustainable development in a quickly evolving world. Vehicular ad-hoc networks (VANETs) [2] represent a domain of ITS that offers several innovative, reliable and cost-effective applications that can be used in a wide range of services, from safety applications to entertainment for drivers and passengers (internet access, etc.). Due to these applications, advances in communications have played a major role in VANETs [3]. This has allowed the deployment of three different architectures (Figure 1), including hardware and software structures that provide communication between vehicles $(\mathrm{V} 2 \mathrm{~V})$, offering flexible and less expensive communication. In this strategy, the vehicles can communicate directly without infrastructure or any installation on roads. However, due to the highly dynamic mobility involved, the network is faced with frequent disconnection. Communication between vehicles and infrastructure or roadside units (RSUs) (V2I/V2R) is, therefore based on a communication system using RSU infrastructure or a base station installed at the edge of the road. The RSUs are connected via a wireless or wired interface. The use of RSUs has the advantage of providing users with services 
relating to internet access, road traffic and exchanging data between the car, garage or home, for remote diagnostics. Another option is hybrid communication (V2V and V2I), which combines two communication modes (V2V and V2I) in order to achieve effective communication. Many factors need to be taken into account in order to meet the requirements for deploying different types of vehicular applications [4], [5] or VANETs. The network connectivity with/without the use of an RSU varies according to the density of the VANET environment; a high density allows the network to be connected, while a low density has a longer transmission delay and high communication failures. In addition, the Quality Of Service (QoS) requirements for real-time applications include the end-to-end delay and packet transmission rate. For these reasons, the design of an efficient routing protocol that meets all the requirements of a VANET is considered a major challenge. Thus, improving reliability and reducing interference of the system in VANET, need a promising routing protocol which should be evaluated using the standard IEEE $802.11 \mathrm{p}$. There are several survey studies that compare routing protocols in VANETs, but the majority compare only a small selection of protocols and consider only a limited number of performance criteria and metrics, without taking into account the presence of RSUs. The comparison and perception is not relevant and always remain to be discovered by the user. For this reason, the deployment of infrastructure such as RSUs is insufficient in VANETs.

The main goal of this survey is to contribute a better comparison between a variety of known routing protocols from different categories, involving RSUs and two types of traffic in an urban topology. This kind of study can significantly affect daily life, and its requirements motivate our interest. Our choice to study the environment mentioned above arises from the problems faced in terms of communication [4] due to the high mobility, dynamic topology and scalability of a VANET. Moreover, the density of the network varies, including very dense networks in urban areas where communication is interrupted, leading to many conflicts in communication, and there are therefore numerous problems to address. The aim of our comparative study is to select and examine a variety of protocols that can support communication between vehicles and RSUs in a complicated and highly dynamic environment, such as in a VANET. Thus, our review provides researchers with the ability to choose the most relevant and promising protocol for a routing strategy, depending on the communication with RSUs and to achieve a particular QoS.

The remaining of this paper is organized as follows. In Section 2, we review seven possible protocols, and in Section 3, we discuss related works, describing the most popular studies of VANETs. In Section 4, we pay particular attention to communication with RSUs by carrying outa performance evaluation of a realistic mobility model for a VANET. Our performance measures are described in Section 5, while Section 6 presents the experimental results and an analysis. Finally, the conclusion and future works for researchers are presented in Section 7 and 8.

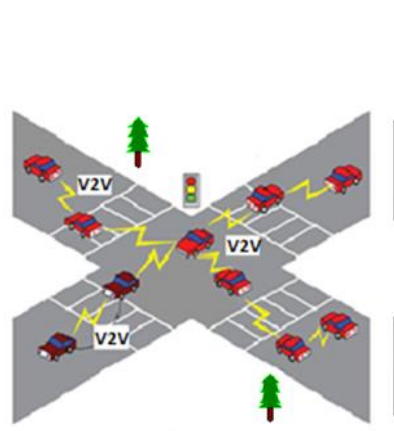

(a)

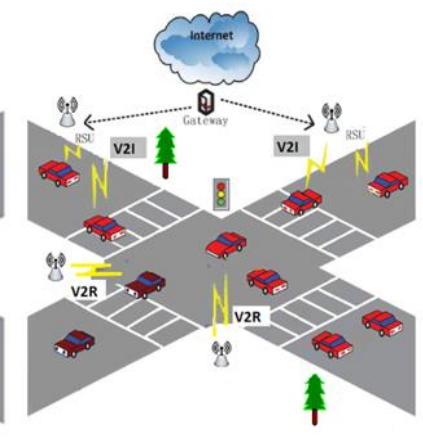

(b)

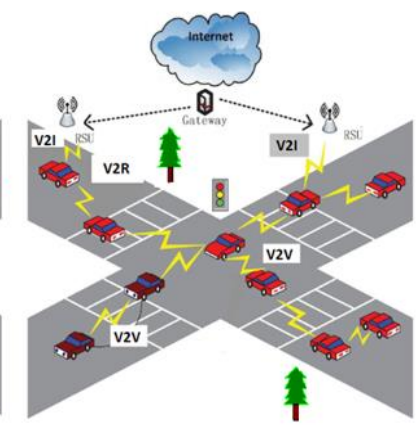

(c)

Figure 1. VANET architecture: (a) vehicle-to-vehicle communication (V2V); (b) vehicle-to-infrastructure or RSU communication (V2I/V2R); (c)hybrid communication ( V2I and V2V) 
International Journal of Computer Networks \& Communications (IJCNC) Vol.12, No.4, July 2020

\section{Overview of Routing Protocols}

In the following paragraphs, we discuss the operating mechanism of routing protocols, the cons and the pros of each one. All protocols are listed with their main advantages and disadvantages in Table 1.

\subsection{Topology-based routing protocols}

The topology-based routing category uses the link information from the routing table to transmit data from the source to the destination. We will present in the next three classifications of this category.

\subsubsection{Proactive routing protocol}

An optimized link-state (OLSR) [6] is an optimized link-state routing protocol. It provides the routes available during the route request due to its proactive nature. Each node exchange topology information's by defining its neighborhood-based on one and two hops, using HELLO messages. From its neighbouring nodes (one hop), it chooses a set of multi-point relays (MPRs) to reach all neighbours at the second hop. OLSR generates three control messages (HELLO, topology control (TC) and multiple interface declaration (MID) messages) to maintain the routing tables, as shown in Figure 2.

Multipath dynamic address routing (MDART) [7] is an advanced extension of the DART protocol [8], which uses the choice of the shortest path in order to respond to changes in topology. It guarantees the transmission of data in several ways, without generating additional communication costs, based on the routing information stored for each node. The discovery of the road and all available routes to the destination in MDART also involve no additional cost.

\subsubsection{Reactive routing protocols (on demand)}

The principle of a reactive protocol is to establish a route on-demand. When a node wishes to transmit a packet, it makes a route request and searches for a path until it arrives at the destination. In our work, we will discuss the AODV, AOMDV and DSR protocols in more detail. A reactive scheme is illustrated in Figure 3.

Ad-hoc on-demand distance vector (AODV) [9] is a protocol that uses an on-demand algorithm from Bellman-Ford. When a source node wants to send a packet, it determines an endto-end route to a destination. The AODV protocol has two well-defined mechanisms: discovery of routes, and maintenance of routes. Route discovery occurs when there is no route to the destination, while route maintenance is responsible for maintaining the route between the source and the destination. The reactive protocol AODV is shown to be suitable for both high and low densities of vehicles in [10], [11]. 
International Journal of Computer Networks \& Communications (IJCNC) Vol.12, No.4, July 2020

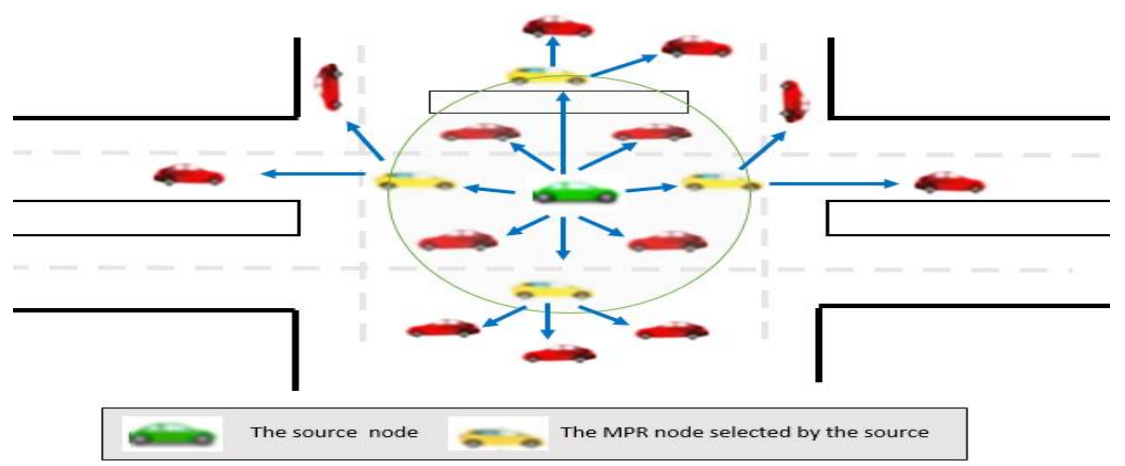

Figure 2. Operating mechanism of protocol OLSR using Multipoint Relays (MPRs)

Table 1 : Pros and Cons of routing protocols

\begin{tabular}{|c|c|c|c|}
\hline Protocol & Classification & Pros & Cons \\
\hline AODV & Reactive & $\begin{array}{l}\text { Updates route to the destination } \\
\text { the use of sequence numbers lead to } \\
\text { eliminate the routing loop } \\
\text { Reduces overloading and processing } \\
\text { of memory } \\
\text { Has a mechanism in case of route } \\
\text { failure }\end{array}$ & $\begin{array}{l}\text { Causes significant delay due } \\
\text { to the route discovery } \\
\text { process } \\
\text { Induces an inconsistency in } \\
\text { the route } \\
\text { Produces heavy overload } \\
\text { due to control messages } \\
\text { Consumes additional } \\
\text { bandwidth }\end{array}$ \\
\hline OLSR & Proactive & $\begin{array}{l}\text { Suitable for high-density networks } \\
\text { Reduces transmission delay of packets } \\
\text { Supports real-time applications }\end{array}$ & $\begin{array}{l}\text { Causes congestion in the } \\
\text { network due to frequent } \\
\text { sending of control packets }\end{array}$ \\
\hline DSR & Reactive & $\begin{array}{l}\text { Includes the routes in the header of the } \\
\text { packets, which eliminates routing } \\
\text { loops } \\
\text { Uses caching when a new route is } \\
\text { discovered }\end{array}$ & $\begin{array}{l}\text { Decreases performance due } \\
\text { to mobility } \\
\text { Causes delay and additional } \\
\text { packet loss in the network }\end{array}$ \\
\hline ZRP & Hybrid & $\begin{array}{l}\text { Designed for large-scale networks } \\
\text { Uses the reactive inter-zone approach } \\
\text { to handle the delay in the network }\end{array}$ & $\begin{array}{l}\text { Highly dynamic topologies } \\
\text { are not supported } \\
\text { The size of the zone is } \\
\text { predefined and cannot be } \\
\text { modified }\end{array}$ \\
\hline MDART & Proactive & $\begin{array}{l}\text { Uses multiple routes between source } \\
\text { and destination to handle topology } \\
\text { changes } \\
\text { Provides protection against road } \\
\text { failure by dividing traffic into a set of } \\
\text { disjoint paths }\end{array}$ & $\begin{array}{l}\text { Produces a high number of } \\
\text { control packets in high- } \\
\text { mobility networks }\end{array}$ \\
\hline AOMDV & Reactive & $\begin{array}{l}\text { Provides effective fault tolerance in a } \\
\text { dynamic network } \\
\text { Manages the charge in the network } \\
\text { and avoids the possibility of } \\
\text { congestion } \\
\text { Maintains connectivity }\end{array}$ & $\begin{array}{l}\text { Induces an increase in } \\
\text { message charges during } \\
\text { route discovery due to } \\
\text { flooding of multiple-node } \\
\text { RREQ messages }\end{array}$ \\
\hline GPSR & Geographical & $\begin{array}{l}\text { Uses greedy forwarding technique to } \\
\text { transmit packets } \\
\text { Suitable for scalability and evolving } \\
\text { networks }\end{array}$ & $\begin{array}{l}\text { Ineffective without GPS } \\
\text { device } \\
\text { Performance degradation } \\
\text { with high mobility }\end{array}$ \\
\hline
\end{tabular}


International Journal of Computer Networks \& Communications (IJCNC) Vol.12, No.4, July 2020

Ad hoc on-demand multipath routing (AOMDV)[12] is an improvement to the protocol AODV that allows for the discovery of several routes between the source and destination. It guarantees a route without a loop, based on sequence numbers. The maintained paths correspond to the maximum value of the sequence number to the destination, and all information about the next hop is kept for the destination. The route discovery process is restarted if all paths fail.

Dynamic source routing (DSR) [13] was designed for ad-hoc multi-hop networks, and forms routes when a source node wants to send a packet to the destination. In addition, it uses the concept of source routing. The two-path mechanisms of AODV are applied in DSR, and each node maintains a cache of routes containing the route leading to the destination nodes.

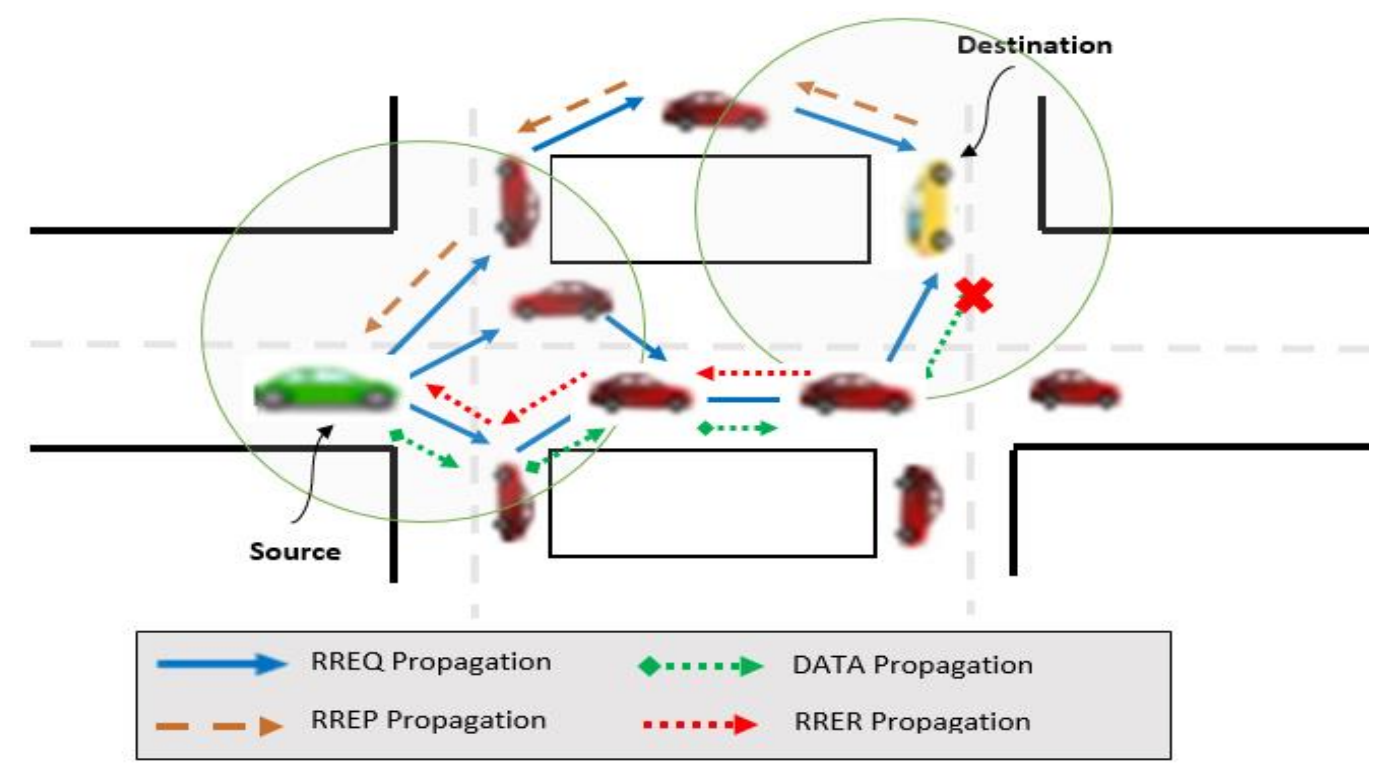

Figure 3. Operating mechanism of Reactive protocol

\subsubsection{Hybrid routing protocols}

The zone routing protocol (ZRP) [14] was one of the first hybrid protocols used in large-scale networks [15]. Route discovery is performed by a limited number of route searches, using interone and intra-zone routing to provide route discovery and route maintenance (see Figure 4). Using reactive routing performed by the inter-zone routing protocol (IERP), which keeps nodes within the zone, it makes a single route request that can result in multiple route responses without overloading resources or loop problems. Proactive routing uses the intra-zone routing protocol (IARP), which updates the status of the network and maintains routes to nodes outside of the routing area. As a result, reactive routing reduces the network overhead and manages the delay caused by reactive routing.

\subsection{Geographic routing protocols}

Greedy perimeter stateless routing (GPSR) [16] based on the geographical position obtained using a positioning system such as GPS. When transmitting packets, each vehicle periodically broadcasts beacon packets to its direct neighbors, and maintains a list containing its own position, the position of its destination and the position of its direct neighbors (one hop), as shown in Figure 5. 
International Journal of Computer Networks \& Communications (IJCNC) Vol.12, No.4, July 2020

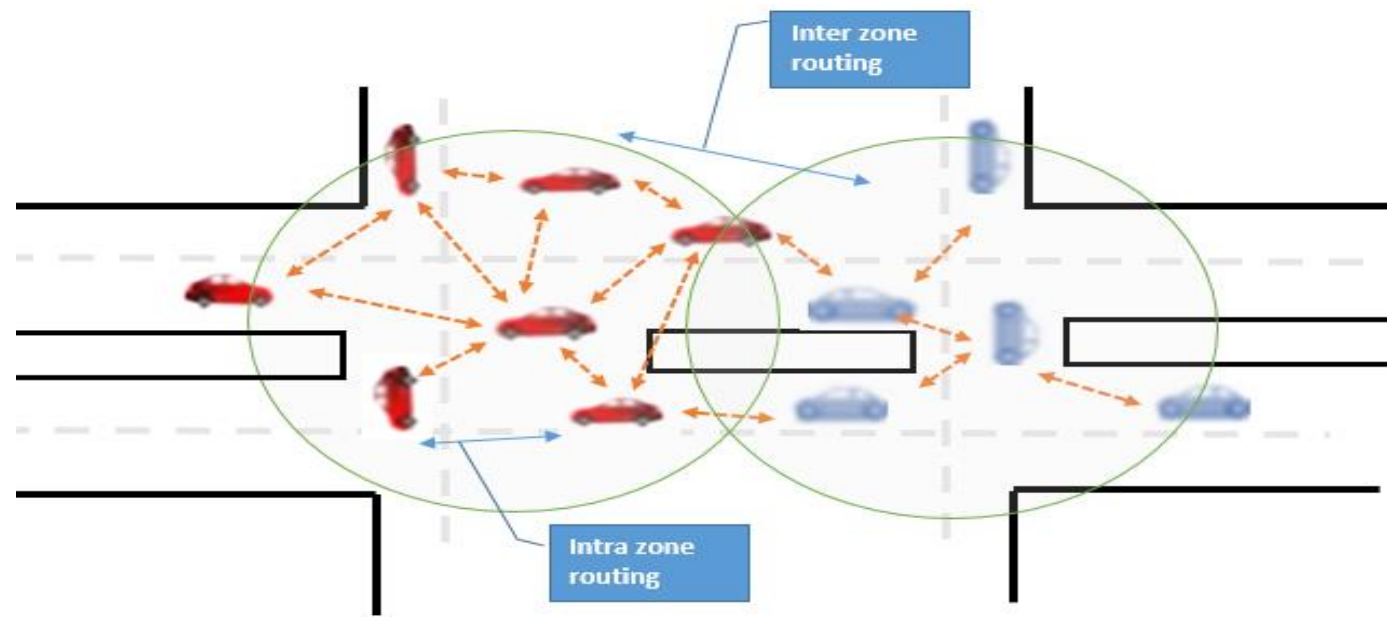

Figure 4. Operating mechanism of hybrid protocol ZRP

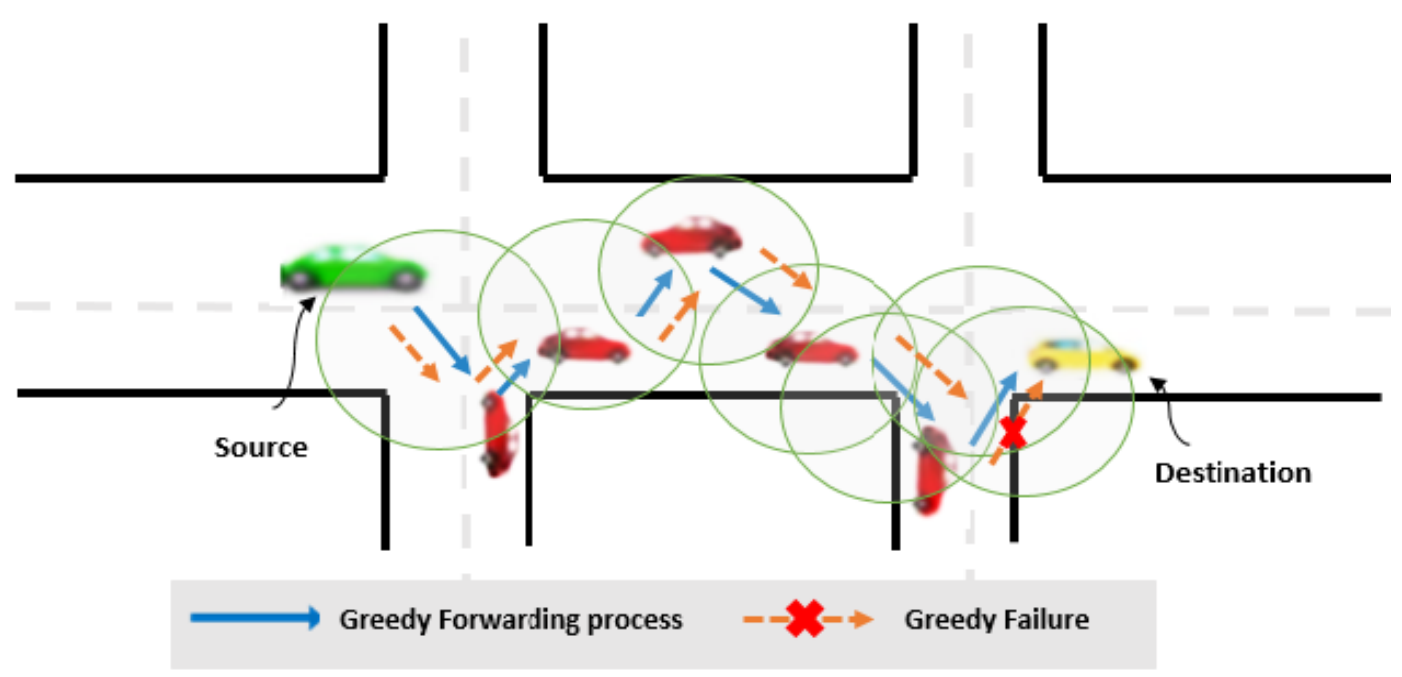

(a)

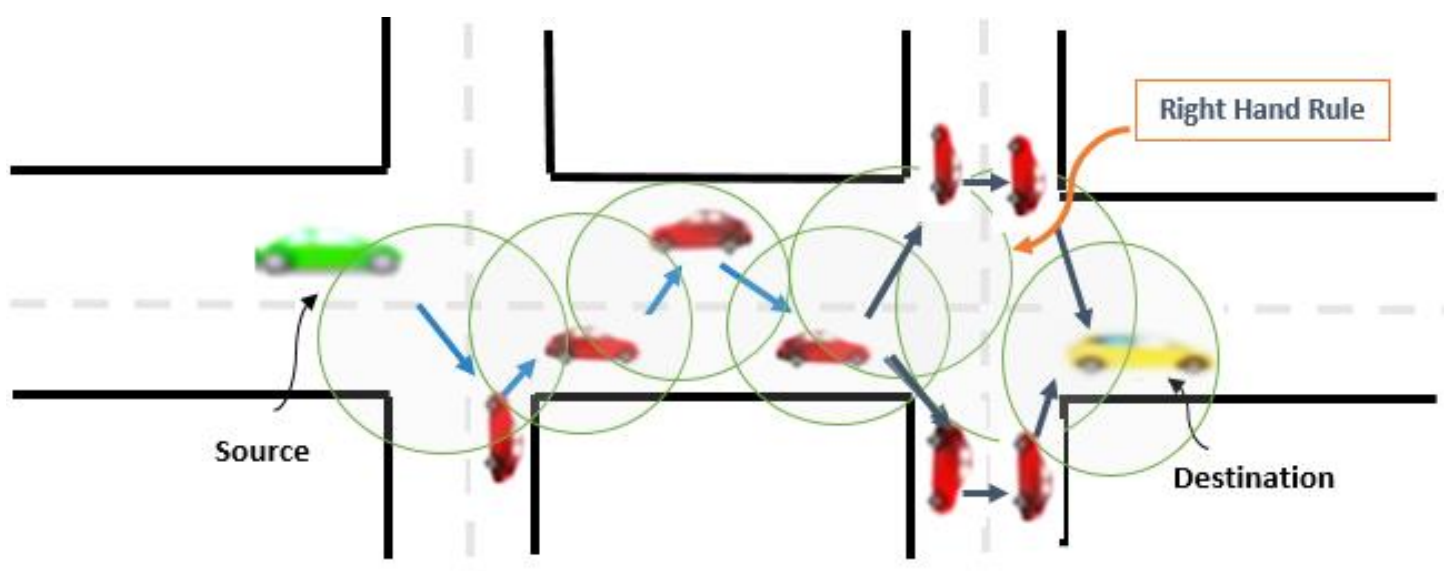

(b)

Figure 5. An exemplary of GPSR functionality: (a) Greedy process and greedy failure (b) perimeter process Right hand Rule 
International Journal of Computer Networks \& Communications (IJCNC) Vol.12, No.4, July 2020

It uses two methods of packet transmission. Greedy forwarding is the first strategy, and this is applied to choose the next optimal vehicle (next hop) that will take over the routing, by selecting the nearest neighbor that is closest to the destination. However, if the transmitter node is a shorter distance from the destination than all its neighbors, the second strategy of perimeter forwarding is applied. This involves using the right-hand rule by routing around the perimeter until a node close to the destination is reached. We assume in our work that the source knows the geographical location of the destination.

\section{RELATED WORKS}

The high mobility and instability of vehicles pose new challenges in VANETs. All routing protocols and perceptions of research aim to enhance the QoS in terms of the packet delivery ratio (PDR), transmission delay and network overhead. However, it is difficult to choose a specific routing protocol that works effectively in all VANET environments. One of the most important surveys was presented in [15]. The authors presented a robust study of VANET dissemination and a specific discussion of performance and QoS requirements. They then carried out a classification and comparison of broadcast protocols by selecting several performance metrics such as the delay, PDR, overhead, throughput, scalability, and network reachability. The authors acknowledged that the mobility of the nodes, frequent topology changes and breaks that characterize VANETs make dissemination a major problem as a research topic. In this context, they discussed the main issues that a researcher may be faced with by examining future challenges and trends that could take advantage of the most effective mechanisms available. They also pointed out that despite existing work, there is still a need for QoS development and optimization, since these studies are based on performance criteria set by researchers according to their topics of interest. The authors of [17] presented a survey of routing mechanisms based on geographical protocols. They classified the different protocols into two categories, namely infrastructure-based and infrastructure-less, the latter of which was classified into basic and advanced routing protocols. In addition, the authors provided an analysis of VANET architectures and various VANET applications. They then presented a comparative study that took into consideration different parameters and variants of greedy forwarding to transmit packets using V2V and V2I approaches. The majority of protocols used GPSR as the base protocol in all simulation scenarios, and the authors suggest that the evaluation of protocols should be performed in these environments. In this context, numerous works have shown many motivations requirements based on the geographic information of vehicles in a VANET. In summary, the overall goal of the various authors of these surveys is to help engineers and researchers to improve vehicle network applications for driver safety and comfort.

Many works have also been dedicated to comparing the performance of routing protocols, considering various performance metrics and parameters such as mobility models. The authors of [18] presented one of these studies, and compared the performance of two protocols OLSR and AODV in urban road scenarios, based on two roads and several intersections. Four metrics are considered: the PDR, the overhead, the throughput, and the E2E delay. The authors used CBR for the data traffic in the two density variation scenarios over transmission ranges of 250 and $500 \mathrm{~m}$. The results showed that the routing costs and delays in both protocols increased with density. It was therefore concluded that the AODV and OLSR protocols are impractical for a VANET with a high density of vehicles. In the same context, the authors of [19] presented a comparative study of the AODV, OLSR and DSR protocols in urban and highway scenarios, in terms of the PDR and E2E delay. Their goals were to achieve robust and easy communication between vehicles for driver safety. The AODV protocol performed well in terms of PDR as function of the density variation. Another study involving a performance evaluation of routing protocols adapted for VANET was presented by the authors of [20]. They evaluated the impacts of the speed and density of vehicles using various metrics. The AODV, AOMDV, DSR, ZRP, DSDV and OLSR 
protocols were simulated using the NS2.35 and SUMO simulator with a CBR data traffic model and various numbers of nodes in a VANET and a MANET. The simulation results showed that the AODV protocol achieved good performance in terms of delay and other performance metrics, unlike in [19], where OLSR outperformed AODV in terms of the E2E delay. The authors concluded that the applicability of MANET routing protocols to a VANET is unsatisfactory, due to the high mobility and low node density. The performance results presented in all these studies provide an adequate overview of the applicability of routing protocols and data integrity to VANETs, and thus could help in designing improvements to the existing protocols.

The studies discussed above [18]-[20] evaluate the performance of VANET networks by considering only the V2V communication mode and CBR data traffic. In contrast, other studies have processed communications using RSU and TCP data traffic. In this context, the authors of[21] present QoS studies of the AODV and DSDV protocols using the two communication modes of V2V and V2I for different RSU relay placements in urban scenarios, based on CBR data traffic. The performance of AODV in all scenarios was better than that of DSDV in terms of the sending, receiving and forwarding throughput and the data loss. The authors observed that the scenario using a strategy with RSUs placed on both sides of the road provided better performance and hence better data transmission. The researchers in [22] analyzed the AODV protocol with a new data transmission scheme for communicating with enhanced RSU. The purpose of this new data transmission scheme was to discover routes in order to reduce overhead and improve QoS. The results showed that the proposed method improved significantly the performance compared to AODV protocol, in terms of the PDR and overhead. The authors of [23] considered two types of data traffics in an urban environment depending on certain metrics. Their analysis of various routing protocols addressed many of the problems of $\mathrm{V} 2 \mathrm{~V}$ communication and will be consistent with future simulation environments for VANETs. The researchers in [24] used TCP with CBR data traffic to evaluate the AOMDV protocol in terms of the PDR, E2E delay, packet loss ratio and throughput. The AOMDV protocol was designed to efficiently transmit data with respect to the AODV protocol in a VANET network. The multipath AOMDV protocol minimized the delay and packet loss ratio and maximized the PDR, while enhancing the throughput. To our knowledge, no attempt has been made to study and evaluate the performance of RSUs with TCP and CBR traffic, in an urban environment; this is a problem of interest for VANETs, since it involves many problems and complications that need to be addressed.

The motivation for carrying out this survey is to bring forth an up-to-date global summary of routing protocols in VANETs, and a performance evaluation of an enormous range of a routing mechanism. Unlike other surveys, our work evaluates and compares the most popular and efficient routing protocols with significant description. To the best of our knowledge, there is a lack of simulation studies or comprehensive research that evaluates and compares routing protocols using IEEE 802.11p technology. Thus, a study of the impact of varying data traffic conditions while exchanging information with RSUs is required. This paper aims is to describe and analyze the performance of seven popular and important routing protocols considering RSUs in an urban area with two types of data traffic and different QoS performance metrics. This analysis can be useful for researches and engineers when developing practical applications and when selecting the most appropriate routing protocols while satisfying the constraints of VANET networks. 
International Journal of Computer Networks \& Communications (IJCNC) Vol.12, No.4, July 2020

\section{Performance Evaluation}

\subsection{Model of mobility}

The vehicle mobility model has been widely used in the literature, due to its ability to provide an accurate model for VANET nodes. According to the authors of [25], the integration of a mobility model can produce significant results. In our work, we use the VanetMobiSim [26] generator, as it provides a high level of realism in our simulation scenarios. Mobility generators can be classified into two types: macroscopic and microscopic. The macroscopic model captures the traffic conditions that influence vehicular traffic, for example the characteristics of a road (one or two-ways, speed, and numbers of lanes), the topology of the road network, traffic lights at intersections and signs, etc. Although this type of simulation is more complex, it is very useful and is necessary when studying the interactions of vehicles with infrastructure. Following [27],we select the mobility model, Intelligent Driver Model with Lane Change model (IDM-LC), which takes into account traffic lights, intersections, vehicle speeds and lane changes, and is therefore the most convenient for our work. Table3 shows the various parameters used on the experiment evaluation in VANET.

\subsection{Parameters of scenarios}

In our simulation, we compare different ad hoc routing protocols in an urban environment using the NS-2 network simulator depending on the type of traffic required by the user (voice, data or video). Which are the file transfer protocol FTP/TCP and the constant bit rate protocol CBR/UDP. A random graph of streets is generated by VanetMobiSim, with a variable number of vehicles and a topology network with nine RSUs. In order to increase the success rate for packet delivery and decrease the average delay, we use IEEE802.11p, which is suitable for a VANET. The main simulation parameters and network characteristics describing the scenario are presented in Table 2. The macroscopic and microscopic mobility model parameters are presented in Tables 3 and 4 [28].

Table 2: Values of the different parameters used in the simulation

\begin{tabular}{l|l}
\hline Parameters used for traffic model & Value \\
\hline Background traffic & CBR/UDP \\
Number of connections & FTP/TCP \\
Packet size & $10,15,20,25,30,35,40$ \\
Packet rate & 512 packets \\
Simulation time & $300 \mathrm{~s}$ \\
& \\
Simulation parameters and network characteristics & \\
Simulator version & NS-2.35 \\
MAC \& PHY layer protocols & IEEE $802.11 \mathrm{p}$ \\
Transmission range & $250 \mathrm{~m}$ \\
Propagation model & Two-ray ground \\
Type of antenna & OmniAntenna \\
Parameters used for mobility model & \\
Dimensions of the simulation area & \\
Number of vehicles & $1000 \mathrm{x} 1000 \mathrm{~m}^{2}$ \\
Mobility model & 50 vehicles \\
Traffic direction & IDM-LC \\
\hline
\end{tabular}


Table 3: Parameters of the microscopic mobility model

\begin{tabular}{l|ll}
\hline Parameter & Description & Value \\
\hline I & Length of vehicles & $5 \mathrm{~m}$ \\
A & Maximum acceleration & $0.6 \mathrm{~m} / \mathrm{s}^{2}$ \\
B & Deceleration & $0.5 \mathrm{~m} / \mathrm{s}^{2}$ \\
Ath & Threshold of acceleration & $0.2 \mathrm{~m} / \mathrm{s}^{2}$ \\
S0 & Jam distance & $2 \mathrm{~m}$ \\
T & Safety distance & $1.5 \mathrm{~s}$ \\
P & Politeness & $0.5 \mathrm{~s}$ \\
minstay & Min stay duration of node & $5 \mathrm{~s}$ \\
& & \\
maxstay & Max stay duration of node & $30 \mathrm{~s}$ \\
\hline
\end{tabular}

Table 4: Parameters of the macroscopic mobility model

\begin{tabular}{l|llll}
\hline Clusters & $\begin{array}{l}\text { Obstacles by } \\
100 \times 100 \mathrm{~m}^{2}\end{array}$ & $\begin{array}{l}\text { Clusters in the } \\
\text { simulation zone } \\
(\%)\end{array}$ & $\begin{array}{l}\text { cluster density } \\
\left(1000 \times 1000 \mathrm{~m}^{2}\right)\end{array}$ & $\begin{array}{l}\text { Speed } \\
(\mathrm{km} / \mathrm{h})\end{array}$ \\
\hline Downtown & 2 & 10 & 4 & 60 \\
Residential & 5 & 40 & 4 & 40 \\
Suburban & 1 & 50 & 4 & 100 \\
\hline
\end{tabular}

\section{Performance Measures}

PDR is the ratio between the total number of packets received and those sent by the source. This metric reveals the reliability and efficiency of the protocol in terms of guaranteeing the delivery of packets. The following equation is used to calculate the PDR:

$$
\mathrm{PDR}=\frac{\sum \text { ReceivedPackets }}{\sum \text { SentPackets }}
$$

End-to-end delay (E2E or EED) is the average time taken by the data packets between the source and the destination. E2E is a metric that needs to be improved with the shortest delay. It is also important in security-related applications. The following equation is used to calculate E2E:

$$
\begin{aligned}
& \mathrm{EED}=\underline{\sum_{i=0}^{n} \text { TimePacket Rcv }_{i}-\text { TimePacketSent }_{i}} \\
& \text { NumberOfPacketsReceived }
\end{aligned}
$$

Packet delay variation (jitter) is the average difference between the delays of twosuccessive packets $(\mathrm{X}$ and $\mathrm{Y})$ received by a node and belonging to the same data stream. It is calculated as follows:

$$
\text { Jitter }=\sum(\text { DelayOfPacket } X-\text { DelayOfPacket } Y)
$$


International Journal of Computer Networks \& Communications (IJCNC) Vol.12, No.4, July 2020

RoutingCost is the amount of information in the network required to receive a packet of data. It is calculated based on the number of bytes for routing packets, including the transmission of routing packets and control packets, divided by the sum of the number of received data packets.It is calculated as follows:

$$
\text { Routing Cost }=\frac{\sum \text { TransmittedRoutingPackets }}{\sum \text { ReceivedDataPackets }}
$$

Efficiency it is an important metrics to show the amount of routing packets sent efficiently to the target. It is calculated as follows:

$$
\text { Efficiency }=\frac{\sum \text { SentPackets }}{\sum \text { TransmittedRoutingPacket }+\sum \text { DataDelivered }}
$$

\section{EXPERIMENTAL RESULTS}

\subsubsection{Measurement of PDR}

In this section, we study the PDR for CBR/TCP data traffic with RSUs. The traffic load increases due to the high probability of collisions, and therefore, the number of delivered packets decreases. In a simple scenario where a node wants to generate a packet, the routing protocol will find a route to the destination via the discovery mechanism. However, if the MAC layer is unable to transfer a packet because there are no alternative nodes, the packet will be lost.

Figures $6 \mathrm{a}$ and $6 \mathrm{~b}$ show that the best results are obtained with the reactive protocols (AODV and AOMDV), since these have the capacity to maintain the route when sending data. From the same figure, we can see that the use of RSUs leads to a higher PDR for the routing protocol in Figure $6 \mathrm{~b}$ than in Figure 6a. A lower rate is seen for the DSR protocol in the scenario without RSUs, since as mentioned in Section 2; it is unable to repair broken links locally. Better performance is achieved for communication between RSUs and vehicles for a low number of CBR connections. DSR uses an alternative route, as it can store more than one path in the cache. In addition, the GPSR protocol achieves better performance than the other two protocols, due to its routing method, which involves finding the neighbour with the lowest distance to the destination and accurate location information, which is an important factor in achieving a high PDR. The proactive protocol MDART achieves a lower performance than the reactive protocols in both scenarios (with and without RSUs), as the congestion caused by broadcasting RREQ packets may lead to loss of packets. OLSR is the best option, since it can reduce the retransmission of data packets and maintain the best possible path, thus reducing the congestion and the overload. MDART achieved the weakest results, showing a significant decline and then stabilization as a function of CBR connections. In scenarios with RSUs, the proactive protocols obtained a higher PDR than in scenarios without RSUs. The OLSR protocol achieved $85 \%$ for a low number of connections, and $49 \%$ for 40 connections, while the hybrid protocol ZRP decreased progressively in both scenarios and is an inefficient choice for a highly dynamic topology such as a VANET.

From Figure $6 \mathrm{c}$ and $6 \mathrm{~d}$, we have the best results are those for the reactive protocols AODV, AOMDV and DSR in the scenario using RSUs. This is due to their capacity to build routing 
International Journal of Computer Networks \& Communications (IJCNC) Vol.12, No.4, July 2020

information and to maintain a route when it is required to send data. The DSR protocol gives the best performance, as it can store more than one path in the cache. All routing protocols perform well, with a rate greater than $97 \%$ for all numbers of TCP connections. As mentioned in Section 4, the TCP ensure the data delivery from the source to the destination. Thus, the performance of the different routing protocols in both scenarios is effective. The use of RSUs with TCP traffic provides good performance and a generally high connection load, and the rate exceeds $98 \%$ for the geographic protocol GPSR, the proactive protocols OLSR, MDART, the reactive protocols AODV, AOMDV, DSR and the hybrid ZRP protocol. The scenario without RSUs gives low performance for the OLSR, AODV, and ZRP protocols compared to the other protocols in the same scenario. The communication between vehicles and RSUs therefore achieves good performance using TCP data traffic, because it is easier to send a packet to a fixed target such as an RSU than a moving destination. In addition, the more coverage areas increase in the network, the number of vehicles and RSUs will communicate with each other and the possibility of one vehicle being in the coverage area of another is high. Hence, the transmission of packets from the source to the destination will be reliable.

\subsubsection{Measurement of delay}

The total delay includes the delays caused by buffering during transmission at the MAC layer, congestion and route discovery. In fact, the packets generated by the vehicles are not all sent to their destinations. For that, the vehicles put them in the queue. Thus, the performance of each protocol is generated based on its own strategy. As shown in Figure7a and b, the position-based routing protocol GPSR performs better than the reactive protocol DSR and the hybrid protocol ZRP. The effectiveness of GPSR in different traffic scenarios can be explained by its use of neighbour nodes in the next-hop forwarding node. This minimizes the number of hops, hence the time taken to transmit the packet to the target node is reduced. All routing protocols exhibit good performance in both scenarios. We can see that GPSR is the most efficient option in the scenario with RSUs. The reactive protocols achieve better performance in the scenario with RSUs than without, and give better discovered quickly with the presence of RSUs along the route. The proactive protocol OLSR produces a relatively low delay, while the opposite is true for the DSR and ZRP protocols.

As shown in Figure $8 \mathrm{a}$ and $\mathrm{b}$, the routing protocols perform well in both the V2V and V2R communication scenarios in terms of the E2E delay, except for the case of GPSR; this gives a longer delay, since when a decision is made, the vehicle traffic pattern is not considered. Besides, the reactive protocol DSR has a relatively high data delivery delay due to its reactive nature. Regarding the performance of the other protocols with the two modes of communication, we note that the proactive protocols OLSR and MDART, the reactive protocols AODV and AOMDV and the hybrid protocol ZRP perform better than the other mechanisms. These protocols seem to be stable and perform well, with a delay that varies from 15 to $25 \mathrm{~ms}$, as shown in Figure 8a. When RSUs are added, there is unexpected variation between protocols depending on the variation in the TCP data traffic load. AODV, AOMDV, OLSR, MDART and ZRP give good performance with an increase of the TCP connection load. Consequently, these results are better than the remaining schemes. A VANET network consists of vehicles, which act as nodes. These nodes may fail whenever due to software faults and hardware tampering, and this leads to the generation of faulty nodes in the system. 
International Journal of Computer Networks \& Communications (IJCNC) Vol.12, No.4, July 2020

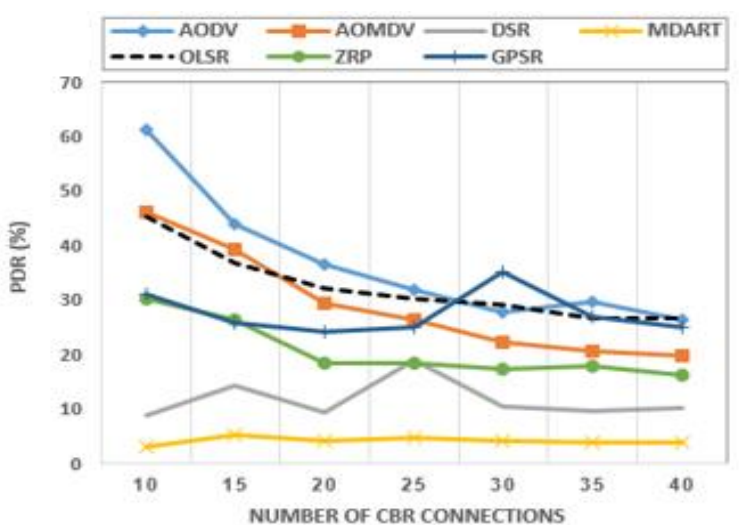

(a)

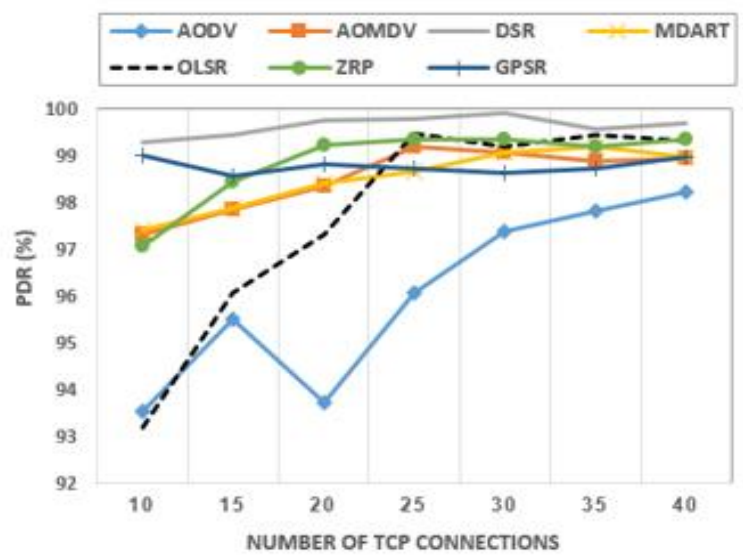

(c)

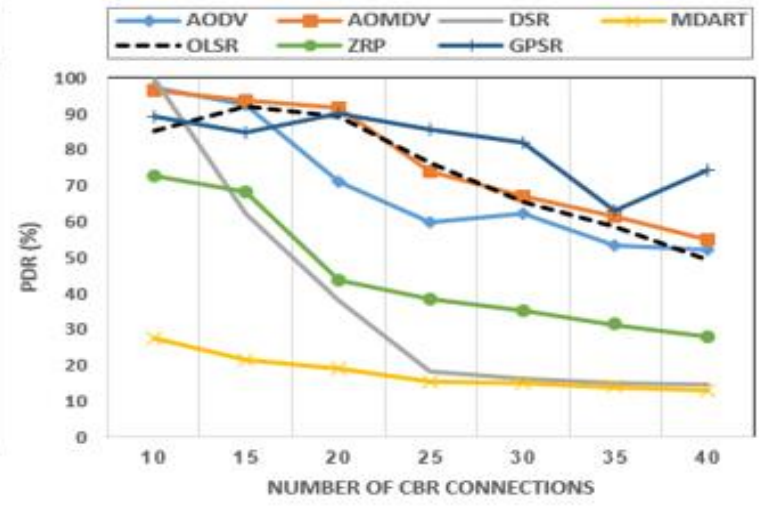

(b)

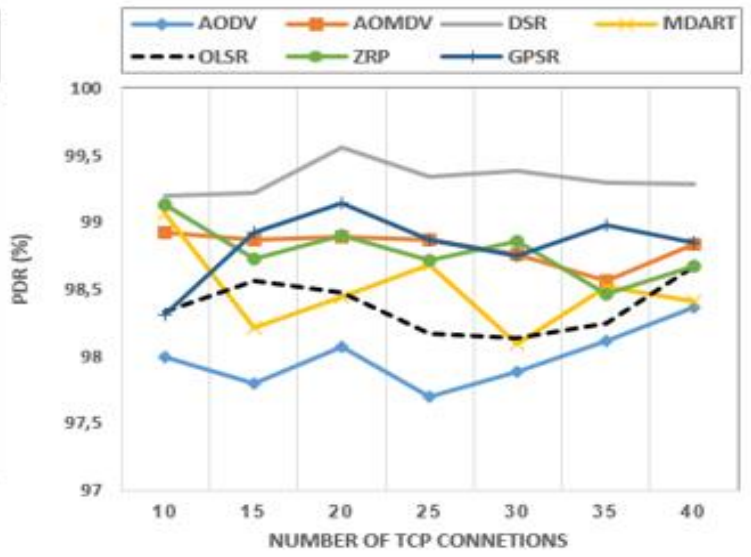

(d)

Figure 6. Packet Delivery Ratio: (a) as function of CBR data traffic load without RSU (b) as function of CBR data traffic load with RSU (c) as function of TCP data traffic load without RSU (d) as function of TCP data traffic load with RSU.

If a vehicle sends data to a vehicle that is faulty at the time of routing, then the data may be dropped and anomalies are obtained. The DSR protocol uses more than one route to transfer data packets from the source node to the destination node, and this causes delay as it does not always use the shortest path to deliver data packets from the source node to the destination node. In DSR, the nodes store the source routes in their caches. When new routes are learned by the nodes, the entries in the cache are updated for these new routes. These anomalies may be because DSR maintains a route cache. 
International Journal of Computer Networks \& Communications (IJCNC) Vol.12, No.4, July 2020

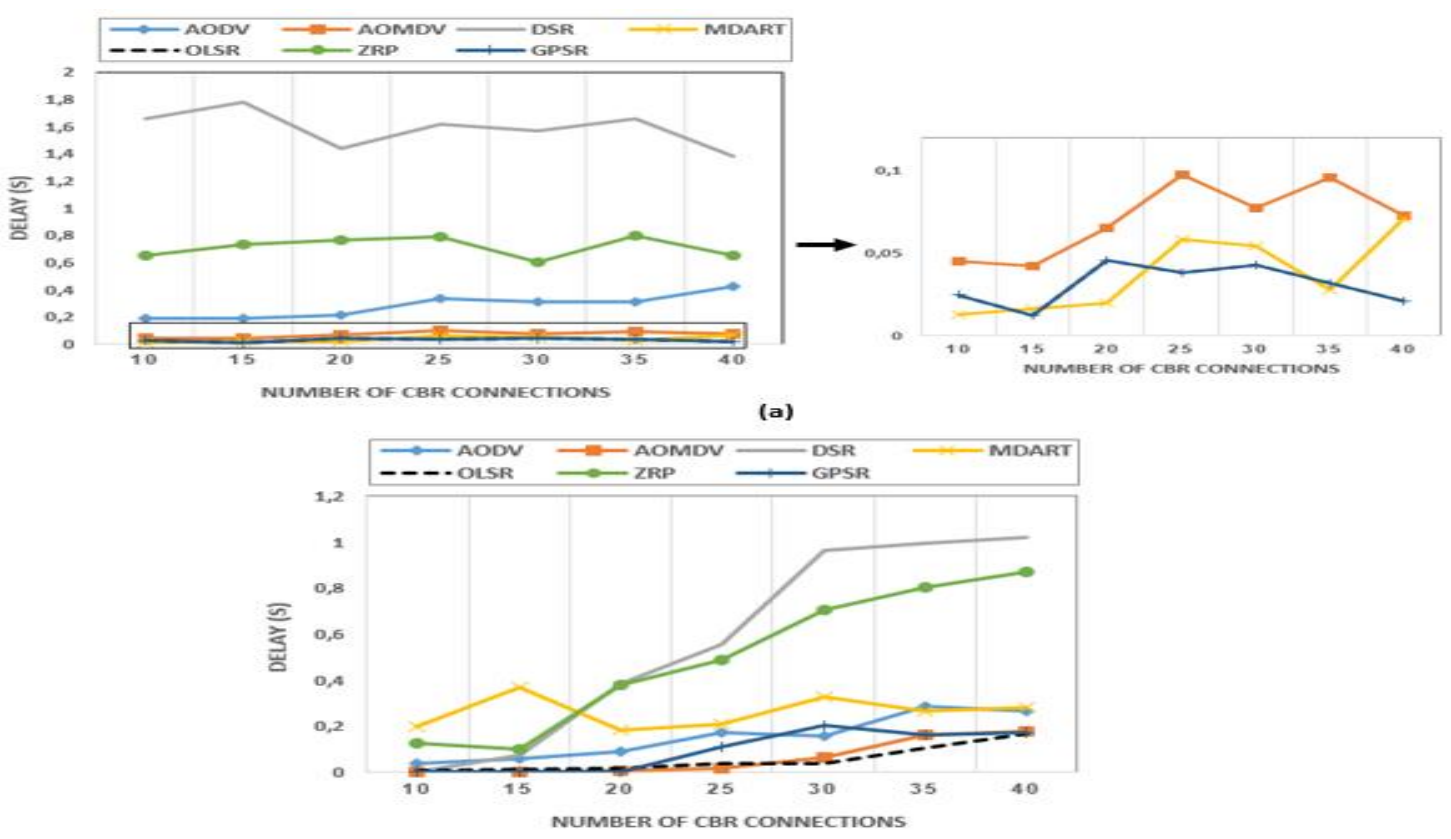

(b)

Figure 7. Delay: (a) as function of CBR data traffic load without RSU (b) as function of CBR data traffic load with RSU

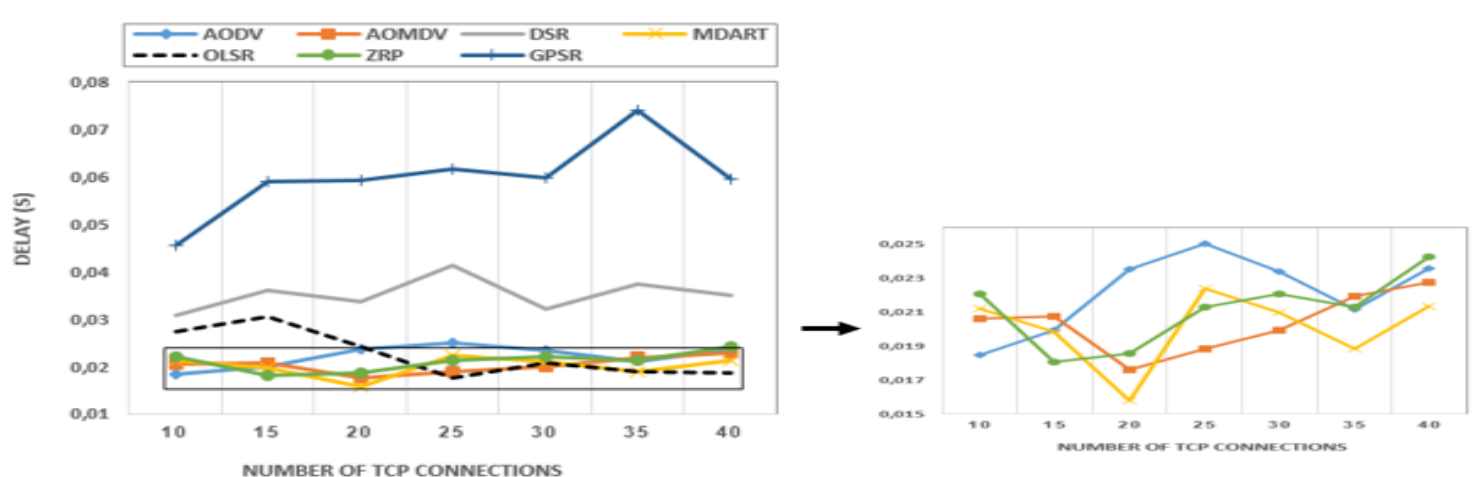

(a)
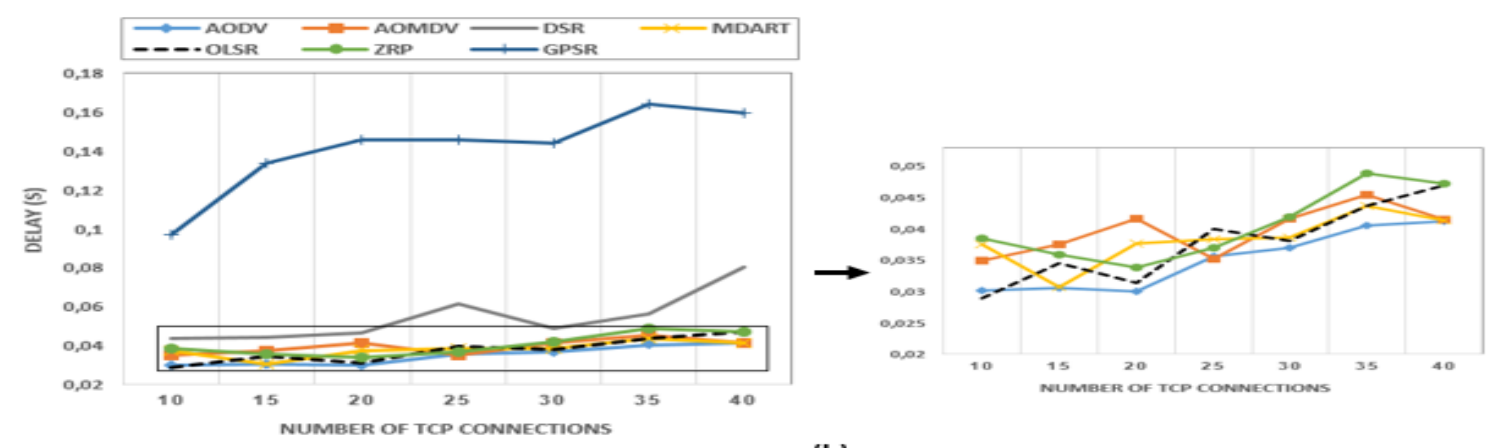

(b)

Figure 8. Delay: (a) as function of TCP data traffic load without RSU (b) as function of TCP data traffic load with RSU 


\subsubsection{Measurement of jitter}

Figure 9 shows the results for the performance of the routing protocols in terms of jitter, which are very similar to those obtained for the E2E delay. The simulated protocols are the most efficient in the scenario with RSUs. We note that the position-based protocol GPSR is the most efficient, and achieves a low data delivery jitter for low CBR data traffic loads. Nevertheless, it isn't meaningful due to its PDR. It should be noted that the majority of protocols show stable performance (except for DSR, ZRP and AODV) with high numbers of connections without RSUs. However, when the CBR load on the network is greater than 20 connections, the hybrid protocol ZRP and the reactive protocol DSR give low results in the scenario with RSUs compared to the geographical protocol. In ZRP, the delay is incurred by the IERP and IARP methods.

We note from Figure 9c and d that all of the protocols give better performance for both modes of communication compared to the CBR connection. The packet delivery delay of the positionbased protocol GPSR is influenced by the TCP data traffic load. GPSR gives poor results compared to DSR in both scenarios. The position of the neighbor node is very likely to change, meaning that the next hop is very likely to move out of communication range, and the delay is then slightly longer. The DSR and AODV protocols give poor performance compared to the proactive protocols and the ZRP hybrid protocol in the scenario with RSUs. The alternation of the values obtained and the intersection between the curves can be explained by the behavior of each protocol in the network the changes in the topology of network over time and the presence of obstacles, which causes the breaking of links. This leads to degrading the performance a function of data traffic load.

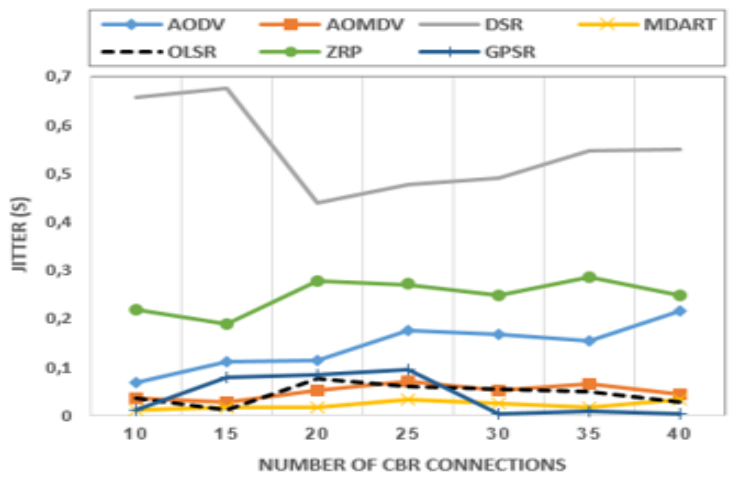

(a)

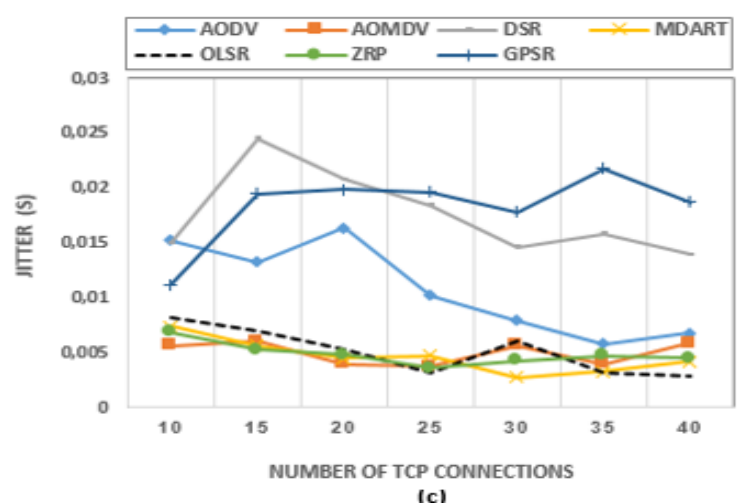

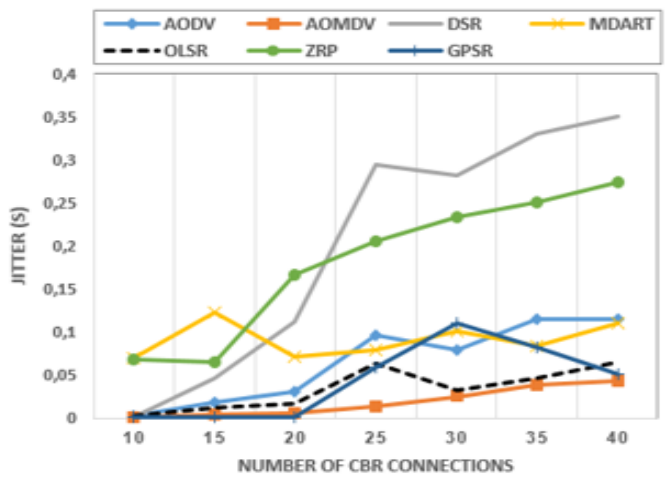

(b)

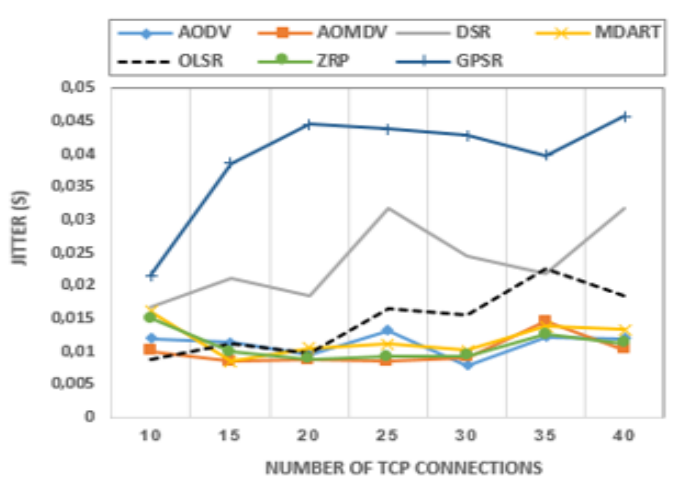

(d)

Figure 9. Jitter: (a) as function of CBR data traffic load without RSU (b) as function of CBR data traffic load with RSU (c) as function of TCP data traffic load without RSU (d) as function of TCP data traffic load with RSU 
International Journal of Computer Networks \& Communications (IJCNC) Vol.12, No.4, July 2020

\subsubsection{Measurement of routing cost}

The routing cost is represented by the ratio between the number of transmitted routing packets and the number of data packets received. At the beginning of this article, we make clear a VANETs as a dynamic networks where vehicles exchange information via wireless links. If we fix the number of nodes, the routing cost decreases as we increase the number of CBR and TCP connections.

The routing costs for the seven protocols for both modes of communication as a function of CBR data traffic load are shown in Figure 10a and b. The routing costs for the various protocols remain almost stable for the two scenarios, since if we increase the number of CBR connections between nodes, the number of control packets remains almost the same. The routing protocols in the scenario with RSUs have a cost that is lower than 35 packets. GPSR, AODV, AOMDV, MDART and OLSR have low overheads, since they do not generate additional RREQ and RREP packets in the network. DSR has the highest cost in both scenarios, followed by the ZRP hybrid protocol.

The impact of the routing cost in the different protocols a function of the variation in the number of TCP connections is illustrated in Figures 11a and $\mathrm{b}$. The number of control packets decreases as the number of connections between nodes and RSUs increases. The best performance is obtained by the GPSR protocol with V2V and V2R communication. A performance evaluation shows that the routing costs in GPSR, OLSR and AOMDV in a network with RSUs are low, and are constant for varying numbers of TCP connections compared with other protocols. The highest values are seen for the DSR reactive protocol and the ZRP hybrid protocol. As mentioned in Section 2, DSR and ZRP are ineffective and rapidly change their topology in a high-mobility network such as a VANET.

Reactive routing protocols such as AODV also suffer when the network is flooded with traffic, at startup time or if there are high levels of traffic between the majority of the nodes in the network. If any other metric than the minimum hop is used, there will be large amounts of overhead traffic. Furthermore, the vehicles in each network are randomly distributed over a real environment in a rectangular distribution, and any relationship failure along the path of the source to reach the target; therefore, we have greater probability of route disconnection when route is long than short ones. The scalability is limited due to a important factors. In fact, in small areas, the need for flooding each RREQ across the network due to the low number of vehicles. Nevertheless, as the network grows, the entire network must be flooded each time a route needs to be discovered by each network node.

\subsubsection{Measurement of efficiency}

Figure 12a and $\mathrm{b}$ show the efficiency of different routing protocols with variation in the CBR data traffic load. The geographical protocol GPSR achieves a rate higher than $80 \%$ with the use of RSUs as a function of the CBR connections. It shows a gradual increase as the number of CBR connections increases. MDART, OLSR and AOMDV also perform well with increasing numbers of connections between nodes and RSUs. The efficiency of the protocols mentioned above becomes almost stable, with a rate greater than 50\%. The reactive protocol AOMDV exceeds 50\% while MDART, GPSR and OLSR reach an efficiency higher than 75\% for 30-40 connections. Moreover, they outperform DSR, AODV and ZRP in both scenarios.

In terms of efficiency a function of TCP connections, Figure $12 \mathrm{c}$ and $\mathrm{d}$ show that the geographical protocol GPSR performs best both with and without RSUs. It achieves a rate of $90 \%$, which remains stable for varying numbers of connections. This allows us to conclude that 
International Journal of Computer Networks \& Communications (IJCNC) Vol.12, No.4, July 2020

GPSR is efficient in dense and dynamic networks. AOMDV and OLSR achieve stable levels of efficiency and better rates than MDART and AODV. AODV shows an increase in efficiency as the traffic increases except for DSR and ZRP, the performance of these protocols reaches a rate of higher than $88 \%$ in the scenario with RSUs, and then stabilizes for high numbers of TCP connections.

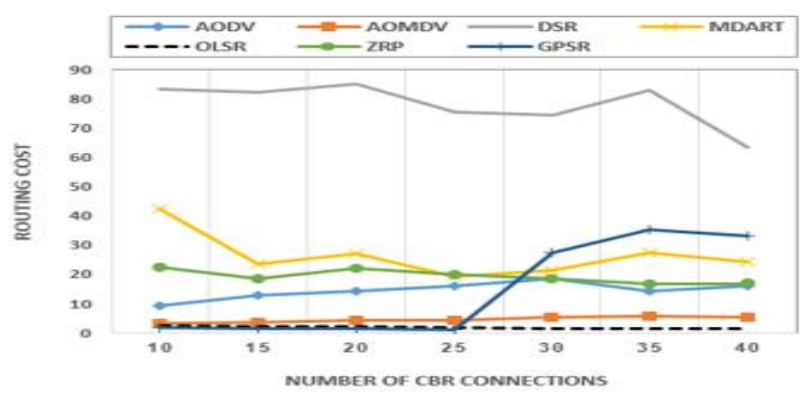

(a)

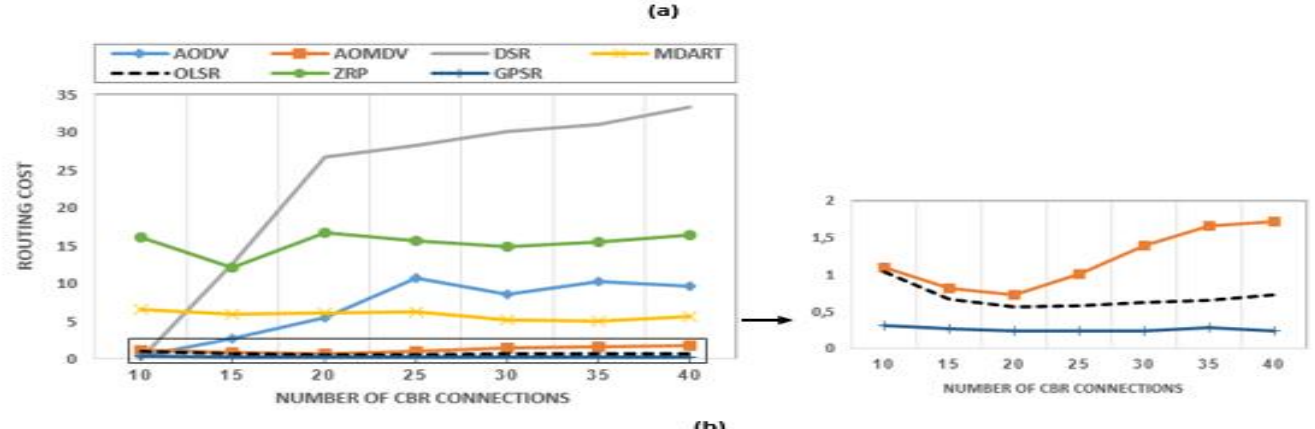

(b)

Figure 10. Routing Cost: (a) as function of CBR data traffic load without RSU (b) as function of CBR data traffic load with RSU
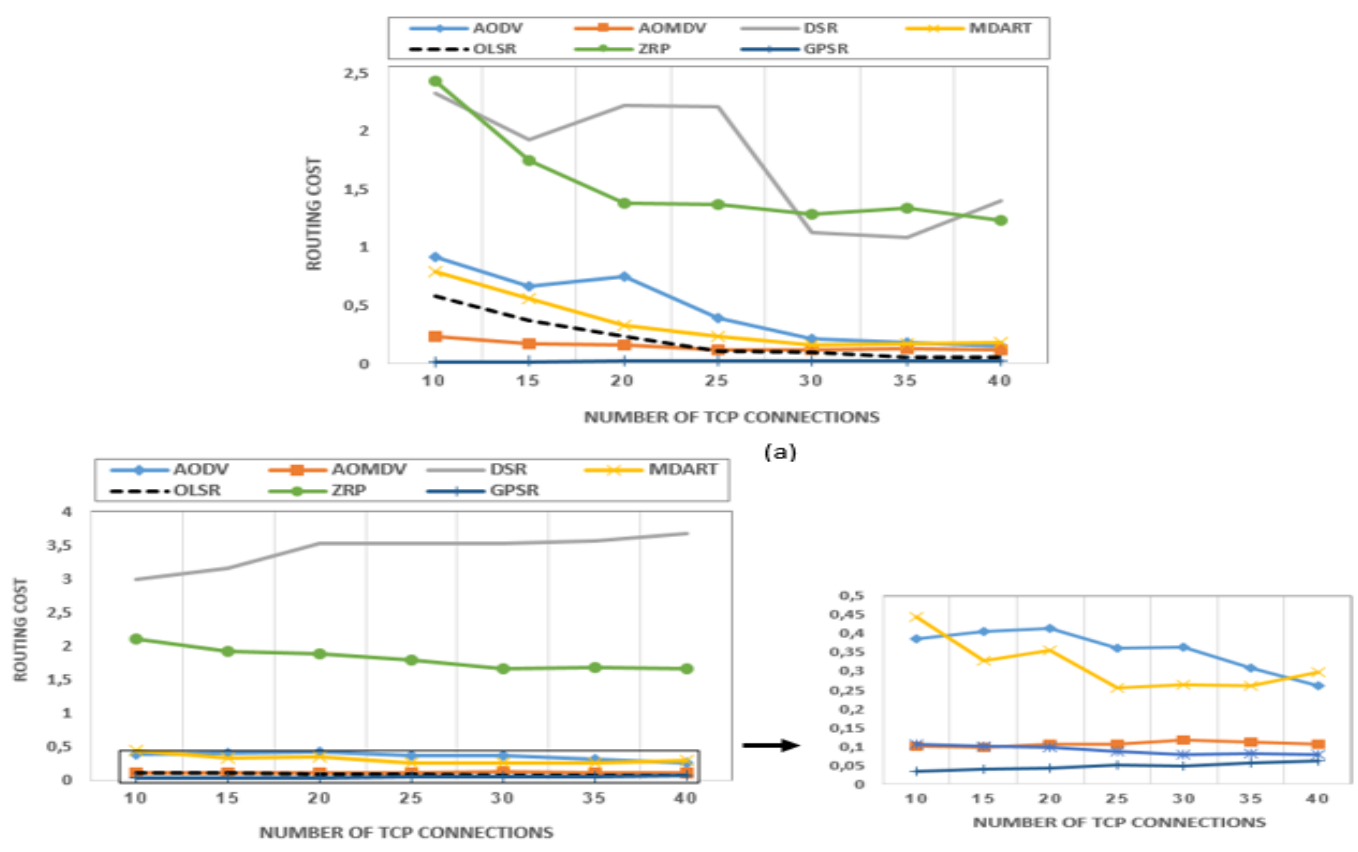

(b)

Figure 11. Routing Cost: (a) as function of TCP data traffic load without RSU (b) as function of TCP data traffic load with RSU 


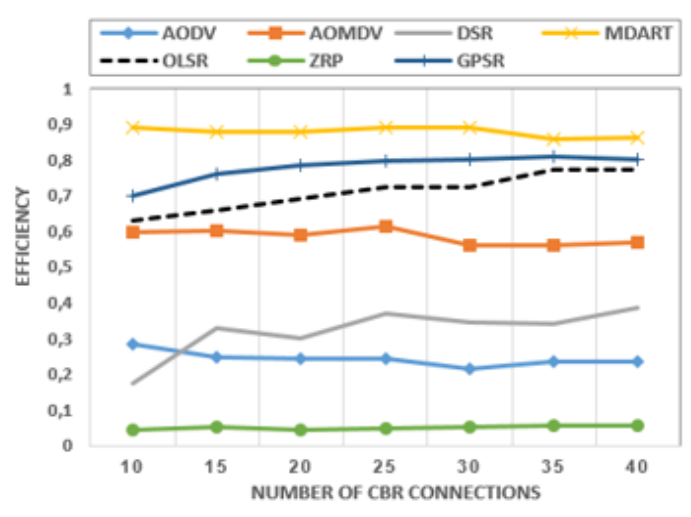

(a)

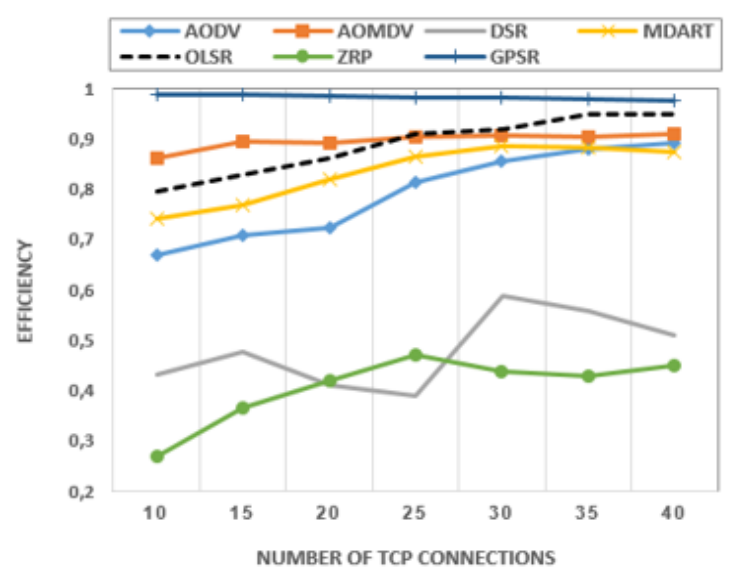

(c)

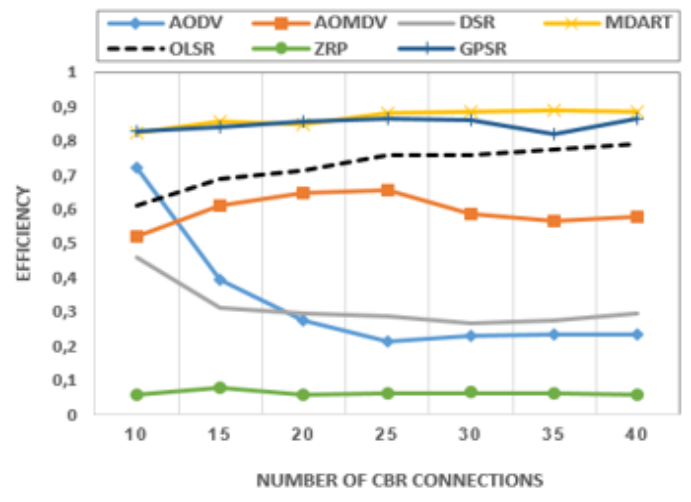

(b)

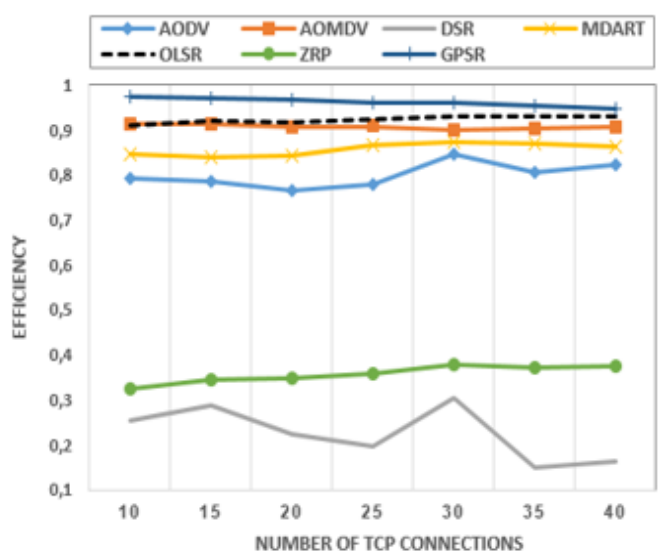

(d)

Figure 12. Efficiency: (a) as function of CBR data traffic load without RSU (b) as function of CBR data traffic load with RSU (c) as function of TCP data traffic load without RSU (d) as function of TCP data traffic load with RSU

\section{DISCUSSION}

Our study presents several important results concerning the evaluation of different routing protocols, in order to analyze the problems encounter in VANETs. Through this study, we can deduce that the use of roadside infrastructure has a strong effect in terms of the QoS compared with communication without infrastructure. We studied an urban environment using RSUs, and summarize our analysis in Table5, which exhibits that the optimum results with regard to minimizing the number of control messages and ensuring the transmission of packets are achieved by the geographic protocol GPSR, for both types of traffic. Adding RSUs to the network allows vehicles to use these fixed infrastructures as they travel along a road, and the use of geographical position gives rise to good performance. However, the use of RSUs degrades the performance for some reactive and proactive protocols. Due to the differences in the mechanism of operation from one protocol to another. The addition of RSUs also generates additional control messages in the network, since the presence of RSUs during the transmission of a data packet requires the generation of several control messages by both the vehicles and the RSUs. As a result, the performance in terms of efficiency will be influenced, since the efficiency depends on the number of control messages (see Section5). It is clear from the results shown in Table 5 that the DSR protocol generates a high routing cost, which causes degradation of the efficiency. 
International Journal of Computer Networks \& Communications (IJCNC) Vol.12, No.4, July 2020

Table 5: Comparison of Seven Routing Protocols Studied in the Urban environment

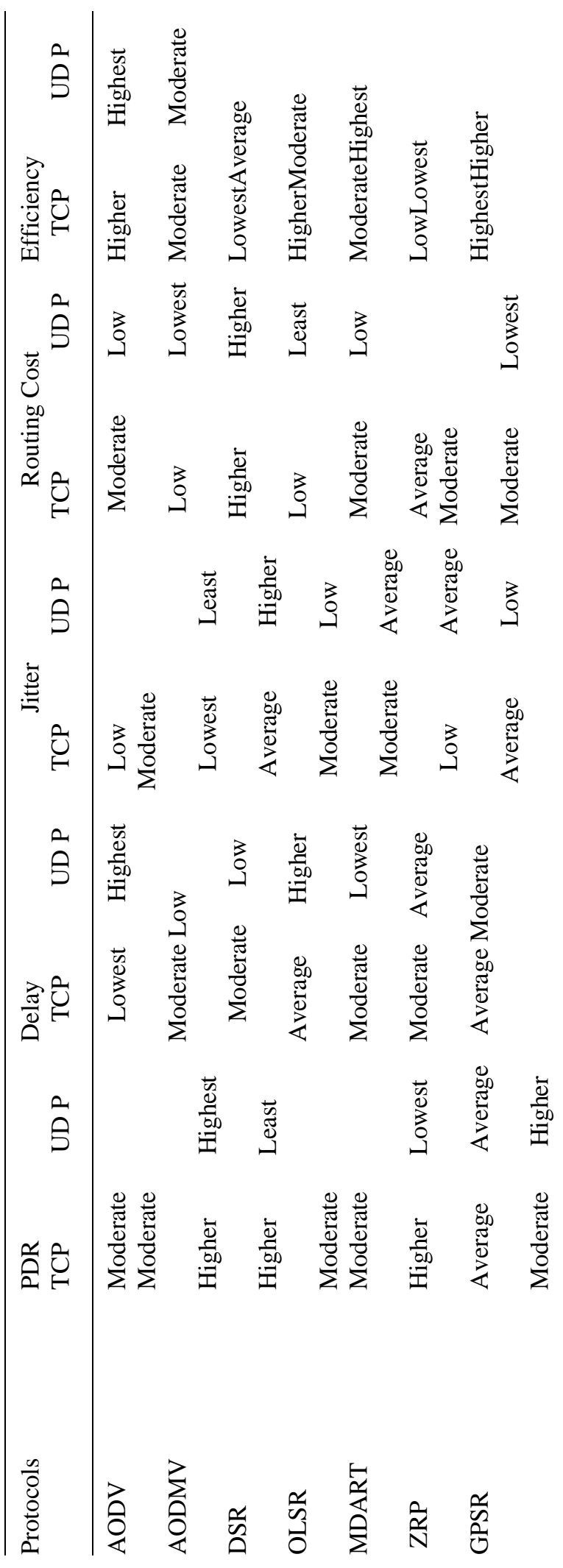


International Journal of Computer Networks \& Communications (IJCNC) Vol.12, No.4, July 2020

The DSR and GPSR protocols incur significant delays for CBR and TCP connection loads, due to high vehicle speeds and loss data packets. The vehicles can therefore be very far apart, leading to long delays. These observations allowed us to realize the importance of routing messages using RSUs along the way. In the concrete case of an urban environment, vehicles tend to drive at different speeds and are faced with several obstacles; they should therefore be able to change the route for each CBR and TCP connection.

\section{Conclusions}

This paper presents a comprehensive research of the various categories of routing protocols in VANET networks, and describes a simulation model based on two types of data traffics. A wide variety of routing protocols are compared in realistic environments under different conditions in terms of their QoS. In view of the problems and difficulties encountered in VANET networks, we studied the response of these routing protocols for V2R communication, using RSU fixed infrastructures and $\mathrm{V} 2 \mathrm{~V}$ communications. The aim of this work is to provide researchers and designers with a better analysis and comparison in order to support the QoS and to give an interesting and practical reference for future works. Based on the analysis of the results, it can be deduced that the use of RSUs has improved the performance of routing protocols compared to communication without infrastructure. The location-based protocol performs well compared to other protocols in both environments. Proactive protocols give better results in terms of time and jitter, due to their proactive natures. In addition, we found that reactive protocols performed well in terms of PDR, routing cost and efficiency, compared to the hybrid ZRP protocol, except for the DSR protocol, which showed performance degradation for some simulation cases. In perspective, we will be moving towards an improvement to geographical versions with other types of traffic and other simulation environments.

\section{CONFLICTS OF INTEREST}

The authors declare no conflict of interest.

\section{REFERENCES}

[1] "ISO 24102-6:2018, Intelligent transport systems - Communications access for land mobiles (CALM) — ITS station management — Part 6: Path and flow management."

[2] Campolo, C., Molinaro, A., \& Scopigno, R. (Eds.) (2015) Vehicular Ad Hoc Networks: Standards, Solutions, and Research. Springer International Publishing.

[3] Campolo, C., Molinaro, A., \& Scopigno, R. (2015) Vehicular Ad Hoc Networks: Standards, Solutions, and Research. Springer.

[4] Da Cunha, F. D., Boukerche, A., Villas, L., Viana, A. C., \& Loureiro, A. A. F. (2014) "Data communication in VANETs: A survey, challenges and applications," INRIA Saclay; INRIA, report.

[5] Vegni, A. M., Cusani, M. B., \& Cusani, R. (2013) "Smart vehicles, technologies and main applications in vehicular ad hoc networks," Veh. Technol. Deploy. Appl.

[6] Jacquet, P. "Optimized link state routing protocol (OLSR)".

[7] Caleffi, M. \& Paura, L. (2011) "M-DART: Multi-path dynamic address routing," Wirel. Commun. Mob. Comput., Vol. 11, No. 3, pp. 392-409.

[8] Ullah, K., Santos, L. M., Ribeiro, J. B., \& Moreira, E. D. S. (2016) "SADP: A lightweight beaconingbased commercial services advertisement protocol for vehicular ad hoc network," in Ad-hoc, Mobile, and Wireless Networks, pp. 279-293.

[9] Das, S. R., Belding-Royer, E. M., \& Perkins, C. E. "Ad hoc on-demand distance vector (AODV) routing."

[10] Haerri, J. (2006) "Performance comparison of AODV and OLSR in VANETs urban environments under realistic mobility patterns". 
International Journal of Computer Networks \& Communications (IJCNC) Vol.12, No.4, July 2020

[11] Ferreiro-Lage, J. A., Gestoso, C. P., Rubiños, O., \& Agelet, F. A. (2009) “Analysis of unicast routing protocols for VANETs," in 2009 Fifth International Conference on Networking and Services, pp. 518-521.

[12] Marina, M. K. \& Das, S. R. (2001) "On-demand multipath distance vector routing in ad hoc networks," in Proceedings of the Ninth International Conference on Network Protocols. ICNP 2001, pp. 14-23.

[13] Johnson, D. B. \& Maltz, D. A. (1996) "Dynamic source routing in ad hoc wireless networks," in Mobile Computing, Imielinski, T., \& Korth, H. F. (Eds.). Boston, MA: Springer US, pp. 153-181.

[14] Haas, Z., Pearlman, M., \& Samar, P. "The zone routing protocol (ZRP) for ad hoc networks."

[15] Mchergui, A., Moulahi, T., Alaya, B., \& Nasri, S. (2017) "A survey and comparative study of QoS aware broadcasting techniques in VANET," Telecommun. Syst., Vol. 66, No. 2, pp. 253-281.

[16] Karp, B. \& Kung, H. T. (2000) "GPSR: Greedy perimeter stateless routing for wireless networks," in Proceedings of the Sixth Annual International Conference on Mobile Computing and Networking, New York, NY, USA, pp. 243-254.

[17] Kumar, S. \& Verma, A. K. (2015) "Position based routing protocols in VANET: A survey," Wirel. Pers. Commun., Vol. 83, pp. 2747-2772.

[18] Jaiswal, R. K. \& Jaidhar, C. D. (2015) "An applicability of AODV and OLSR protocols on IEEE 802.11 p for city road in VANET," in Internet of Things, Smart Spaces, and Next Generation Networks and Systems, pp. 286-298.

[19] Singh, P. K. (2011) "Simulation based analysis of adhoc routing protocol in urban and highway scenario of VANET",

[20] Purohit, K. C., Dimri, S. C., \& Jasola, S. (2017). "Performance evaluation of various MANET routing protocols for adaptability in VANET environment,” Int. J. Syst. Assur. Eng. Manag., Vol. 8, No. 2, pp. 690-702.

[21] Ali, F., Shaikh, F. K., Ansari, A. Q., Mahoto, N. A., \& Felemban, E. (2015) “Comparative analysis of VANET routing protocols: On road side unit placement strategies," Wirel. Pers. Commun., Vol. 85, No. 2, pp. 393-406

[22] Yang, L. \& Liu, H. (2016) "A data transmitting scheme based on improved AODV and RSU-assisted forwarding for large-scale VANET," Wirel. Pers. Commun., Vol. 91, No. 3, pp. 1489-1505.

[23] Smiri, S., Boushaba, A., Abbou, R. B., \& Zahi, A. (2018) "Geographic and topology based routing protocols in vehicular ad-hoc networks: Performance evaluation and QoS analysis," in 2018 International Conference on Intelligent Systems and Computer Vision (ISCV), pp. 1-8

[24] Bondre, V. \& Dorle, S. (2015) "Design and performance evaluation of AOMDV routing protocol for VANET," in 2015 International Conference on Computer, Communication and Control (IC4), pp. 14.

[25] Choffnes, D. R. \& Bustamante, F. E. (2005) “An integrated mobility and traffic model for vehicular wireless networks," in Proceedings of the Second ACM International Workshop on Vehicular Ad Hoc Networks, New York, NY, USA, pp. 69-78.

[26] "VanetMobiSim - Newcom | Institut Eurecom | Politecnico di Torino." [Online]. Available: http://vanet.eurecom.fr/.

[27] Rahman, M. H. \& Rahman, M. U. (2014) "Realistic vehicular mobility impact of FTM, IDM, IDMIM and IDM-LC on VANETs".

[28] Harri, J. \& Fiore, M. (2006) "VanetMobiSim-Vehicular ad hoc network mobility extension to the CanuMobiSim framework," Institut Eurécom Department of Mobile Commu, Vol. 6904, p. 1-19. 